\title{
Increasing the reliability of mining plans by predicting geotechnical instabilities with structural control: case study at a BHP mine, northern Chile
}

\author{
C Roa Minera Escondida, Chile \\ J Calderón Minera Escondida, Chile \\ R Castellón TIMining, Chile \\ M Vargas TIMining, Chile
}

\begin{abstract}
Ore extraction at a mine site is carried out according to a mining plan which is essentially a sequence that follows a given throughput and determines how the mining operation itself will be conducted in terms of time and space, in order to maximise the long-term business value. The continuity of a mining plan can be disrupted by unforeseen geotechnical instabilities that can cause accidents, render roads or areas unusable and damage equipment. This can prevent the operation from complying with the mining plan by having to consider alternative haulage roads, having less equipment available for extraction or having to mine unplanned volumes and grades for a period.
\end{abstract}

This paper describes the results of implementing a geotechnical instability prediction process at a BHP site in northern Chile. This tool allows to identify potential geo-referenced instabilities in real and design topographies located in areas scheduled on the short-term mining plan.

By identifying potential instabilities, the geotechnical team has been able to find plane, wedge and polyhedral block failures in the design of lower benches, involving from one to four benches. In this case study, a high persistence wedge of 500,000 $t$ and four blocks of 200 to 17,000 thave been identified, and the team has been able to warn the planning and operational department so that they could take preventive mitigation or remediation measures, such as: (1) considering changes in the design to prevent instability formation, (2) removing the instability bench by bench to prevent falling, and (3) recommending the operation team to make changes to the blasting schedule in the area, in order to blast the instability when creating the bench.

The geotechnical department has been using these measures to reduce the number of events involving unplanned fall of instabilities, which in turn will limit operational disruptions that cause delays in the mining plan and will improve the chances of carrying out the plan as scheduled.

Keywords: instabilities prediction, geolocalised, mining plan, operational continuity

\section{Introduction}

The extraction of material at a mining operation is carried out according to a mining plan that describes where and when materials, such as ore or waste rock, will be extracted. The extraction of waste rock is for the purpose of making room for the areas containing ore (Lane 1988). Therefore, an optimal extraction sequence is established in order to maximise the business value in the long-term (Dimitrakopoulos et al. 2002). Unforeseen deviations from the planned mining sequence, then, represents a loss of business value.

The continuity of operations may be disrupted by several factors, thus impacting compliance with the mine plan. For example, natural disasters, such as earthquakes or floods can cause landslides that leave areas of the mine inaccessible for a long time. Accidents affecting both people and equipment, prevent the continuity of the scheduled tasks. Poor road maintenance, development of intermediate ramps, or cleaning of small 
landslides also cause delays in the mining plan. Lastly, geomechanical factors, such as landslides due to deformation of the rock mass over time or falling of instabilities or blocks formed by structural planes (Goodman \& Shi 1985) also cause delays in the mining plan due to the clean-up work required to allow equipment to continue operating both safely and efficiently.

This paper describes the results of the use of TIMining's Tangram software (TIMining Tangram 2019) in Minera Escondida, Chile. This tool supports the timely detection of instabilities formed by structural planes, or rather, instabilities with structural control. Using this tool, it is possible to analyse both actual and design surfaces where mining will take place, in order to predict where instabilities will be formed and to be able to take preventive measures.

Examples of such preventive measures are the bench-to-bench removal of an instability as the slope is being built to prevent it from falling; the removal of an instability in a controlled way using the energy of the blasts, or even changing the slope design (bench angle or berm width) in the affected zone to prevent the instability from developing or to contain it in the lower berm in case of falling. Being able to predict where instabilities with structural control will develop in the areas scheduled in the short-term mining plan enables preventive actions to avoid delays in the mining plan or to adjust the short-term mining plan, thus increasing its reliability.

In this work, two geotechnical cases of Minera Escondida are analysed to show how the geotechnical team are dealing with the detection and sizing of potential instabilities in order to prevent accidents and operational discontinuities.

\subsection{Case 1}

An area of one of the Minera Escondida pits has been analysed using TIMining Tangram. According to the geotechnical monitoring system, this area has a high deformation rate, as shown in Figure 1, that triggered an alert.

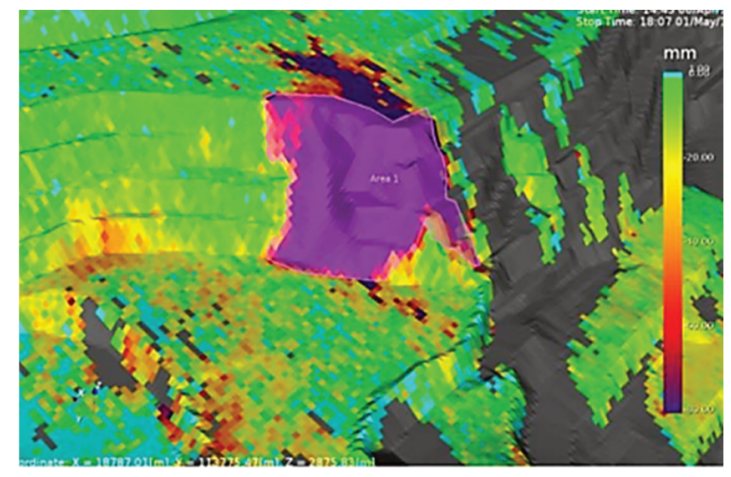

Figure 1 Monitoring system data; higher (purple) and lower (green) deformation rates

After the monitoring system alert, a field inspection was carried out, finding evidence of cracking at the top of the slope, as shown in Figure 2.

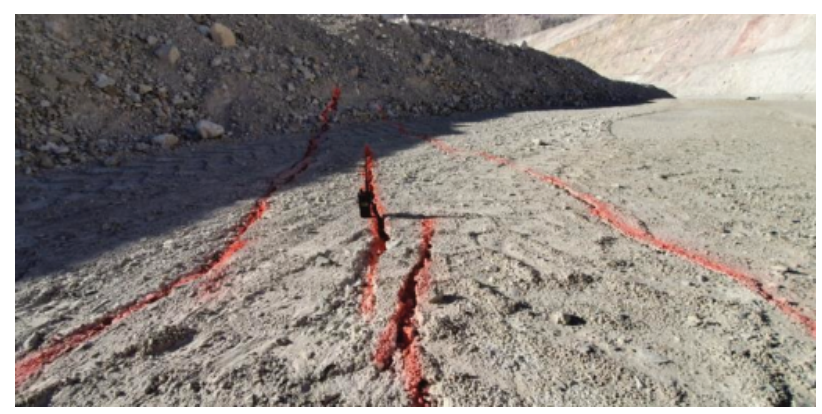

Figure 2 Cracks formed at the top of the slope with a high deformation rate according to the monitoring system 
However, the cause for these cracks was not clear. The purpose of analysing this area was to identify structural systems that could accelerate the deformation rate or facilitate the fall of instabilities in the affected area. A risk mitigation plan would be prepared with these results and the Planning Department would be informed about the actions to be carried out in order to adjust the short-term mining plan.

\section{$1.2 \quad$ Case 2}

This case shows how the geotechnical team of Minera Escondida analysed the short-term design surfaces with the aim to give recommendation of preventive actions to the Planning Department in case there are formed a high number of potential instabilities, or give recommendation of operational actions to the Operation Department in case the instabilities could be removed.

For this objective, in this work two short-term design surfaces are analysed (Figure 3).
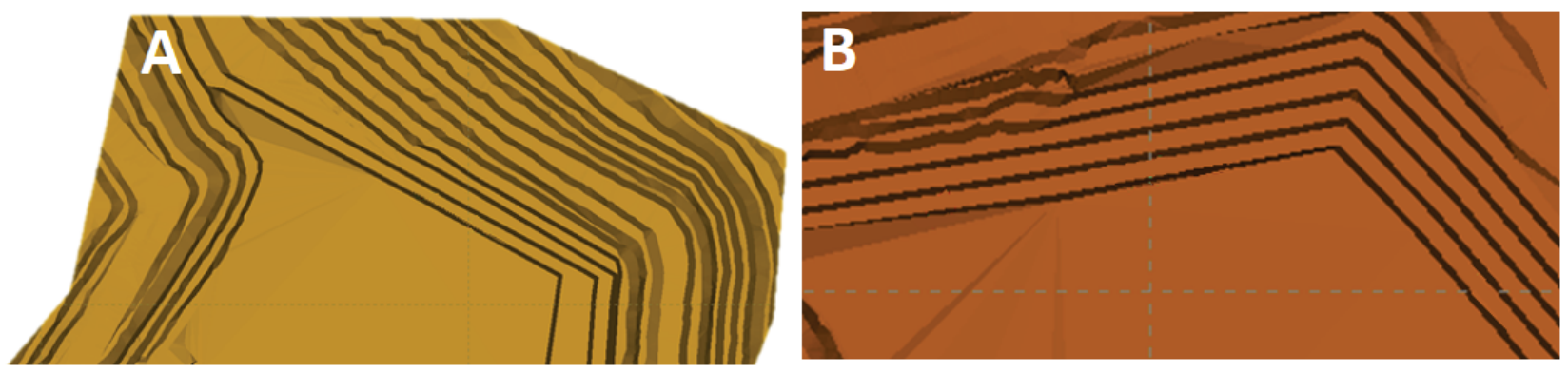

Figure 3 Short-term design surfaces analysed for potential instabilities

\section{Methodology}

TIMining Tangram has been used to analyse different surfaces to find and size potential instabilities. The steps involved are:

1. Loading the surface to be analysed.

2. Loading of the database of structural mappings and fault surfaces.

3. Automatic identification of instabilities to find potential planar, wedge and block (formed by three or more structures) failures.

4. Projection of mapped faults and structures onto short-term design surfaces of lower benches and automatic identification of instabilities, in order to detect potential removable blocks in the short-term.

5. Safety Factor and Failure Probability calculation for each block detected.

6. With these results, the geotechnical team give recommendations of preventive actions to be analysed by the Planning Department, or operational actions to the Operation Department.

\section{Data}

\subsection{Case 1}

The surface, structures, and major/medium fault surfaces existing in the area to be analysed have been loaded in TIMining Tangram, as shown in Figure 4. There are seven major and medium fault surfaces in the site. 


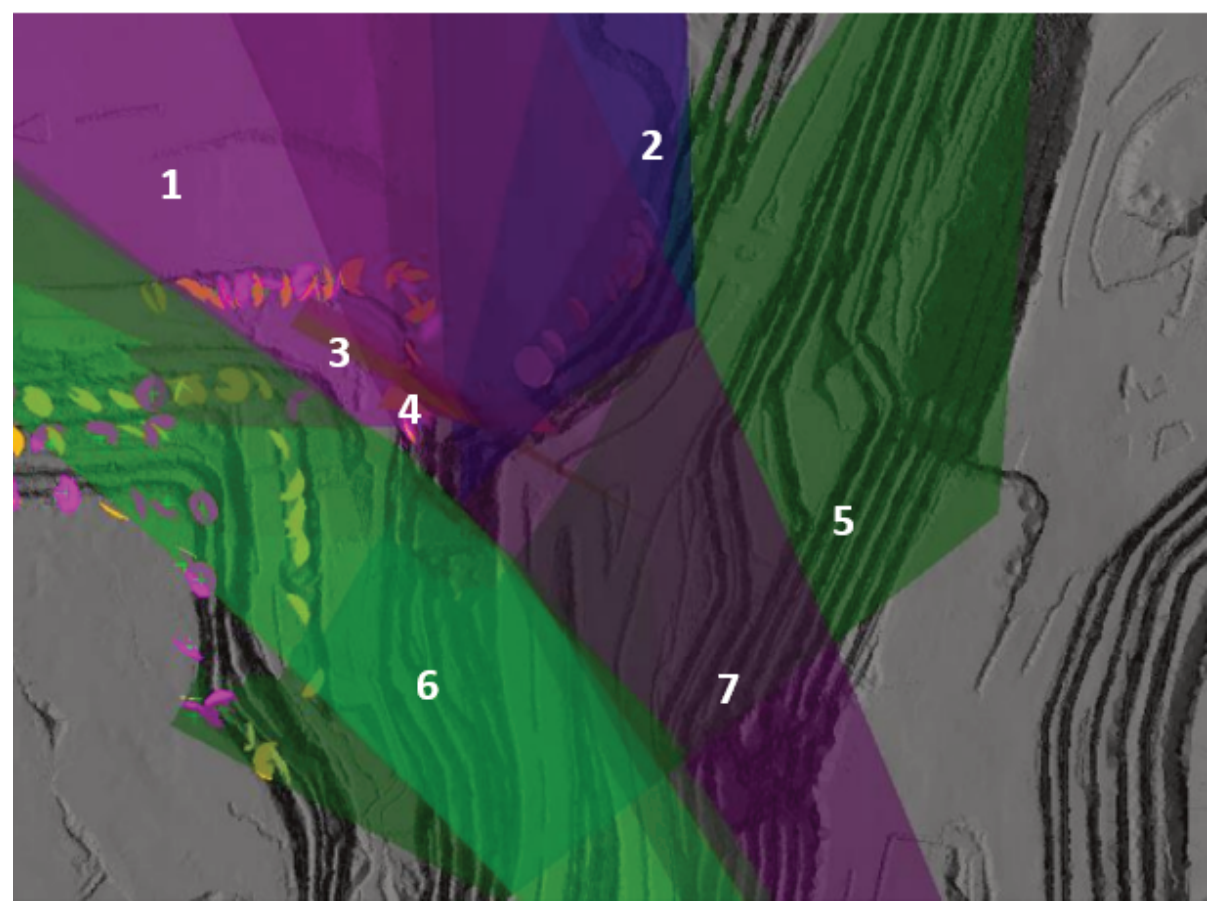

Figure 4 Surface, structures, and major/medium fault surfaces (seven) at the site to be analysed, as loaded in TIMining's Tangram software

Using this data, TIMining Tangram automatically finds instabilities by processing all intersections between structures, fault surfaces and the surface, and between structures and faults.

\subsection{Case 2}

The surface, projected structures, and fault surfaces used to analyse the design surfaces of Figure 3 are shown in Figure 5.

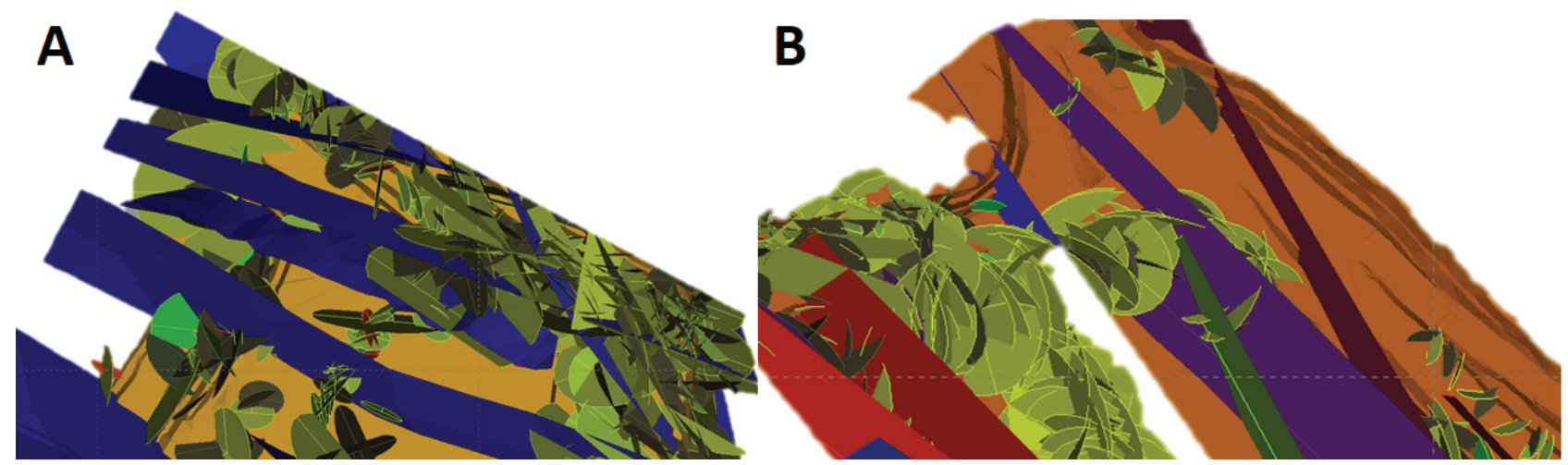

Figure 5 Projected structures, and fault surfaces used to analyse short-term design surface A and B for potential instabilities

\section{$4 \quad$ Results}

\subsection{Case 1}

During the automatic instability detection, a large instability of 500,000 t associated with two main faults was found in the alert zone, as shown in Figure 6. Fault 1 has $40^{\circ} / 298^{\circ}$ dip and dip direction, orientation that is subparallel to the slope and creates the most unfavourable condition. Fault 2 has $75^{\circ} / 30^{\circ}$ dip and dip direction. 


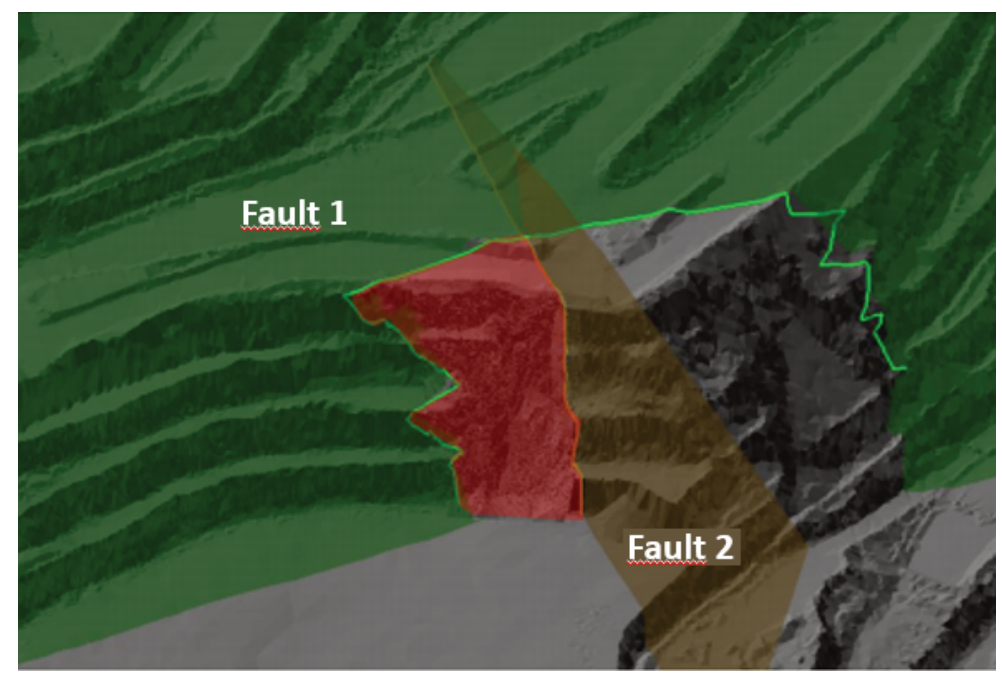

Figure 6 Instability detected in the alert zone (red), limited by Fault 1 and Fault 2

The recommended preventive measure has been to remove the instability by drilling, blasting, loading and hauling of the material in the two upper benches and then loading the material in the two lower benches with supporting equipment. The recommendation includes carrying out preparation works with bulldozers for a significant period before drilling, in order to study the behaviour of the area before the introduction of major equipment, thus evaluating the response of the rock mass based on cracking. The recommendation for drilling has been to use remotely operated equipment.

Removal of the instability took two continuous days, with a total of approximately 12 hours of accumulated work in which 45,000 t were removed without people or equipment-related events to report. Work was interrupted by a new alert from the monitoring system, the risk was communicated, and personnel and equipment were evacuated. No removal work has been conducted afterwards. The status of the area after removal of the 45,000 $\mathrm{t}$ is shown in Figure 7, where the deformation rate decreased with respect to the initial state of the alert.

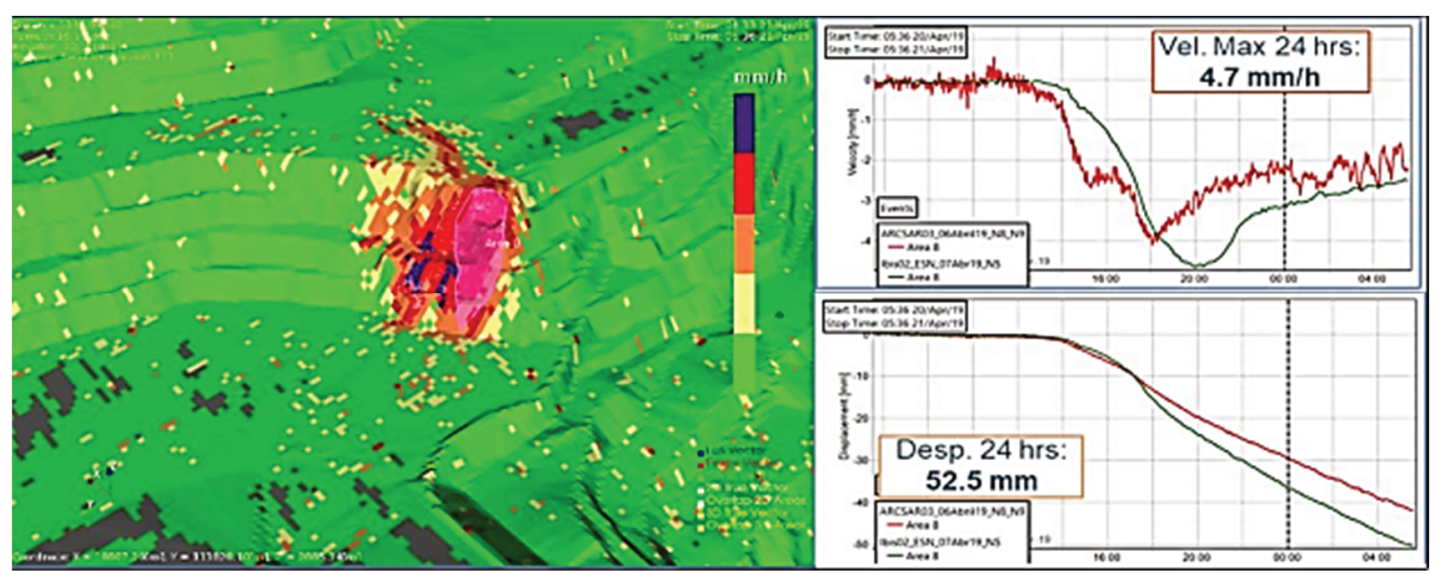

Figure 7 Status of the alert zone after removal of 45,000 t of the instability. Deformation rate has decreased with respect to the initial state; higher (purple) and lower (green) deformation rates

After the second alert, the area has been left to settle without equipment re-entering. The monitoring system shows that the area is currently stabilised, as shown in Figure 8. 


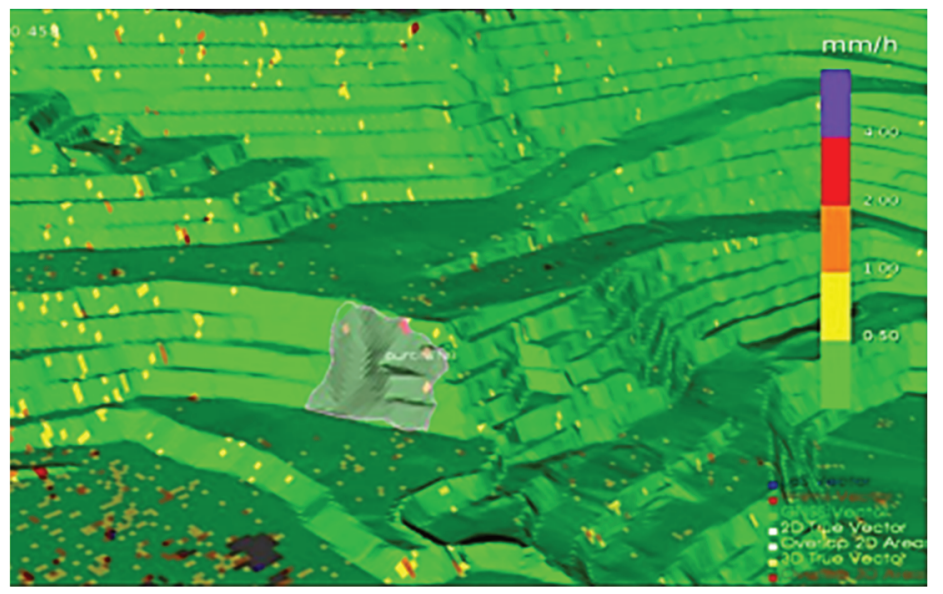

Figure 8 Status of the alert zone. Material has settled and the site is stable. Equipment is not yet allowed to enter the area

After the work to remove materials, a specific work protocol has been created with a focus on drilling and loading operations, due to the possibility that the area becomes active again as a result of ongoing work. This protocol makes the geotechnical department responsible for ongoing inspection, monitoring, communication and definition of limits for people and equipment in the area, while the Mine Planning Department is made responsible for preparing a site-specific mining plan.

\subsection{Case 2}

The intersection of the projected structures and mayor faults with the design surfaces resulted on the detection of 40 to 100 removable blocks approximately on each surface (Figure 9).
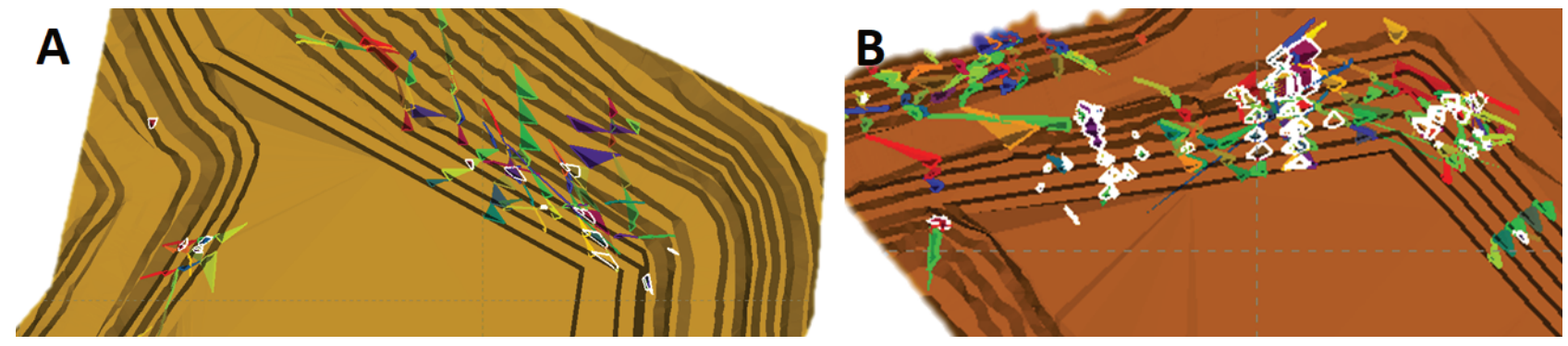

Figure 9 Removable blocks detected on each short-term design surface

Then, the Safety Factor and Probability of Failure of each block was calculated, selecting the blocks which had Safety Factor under 1.5 and Probability of Failure greater than 20\% (Figure 10).

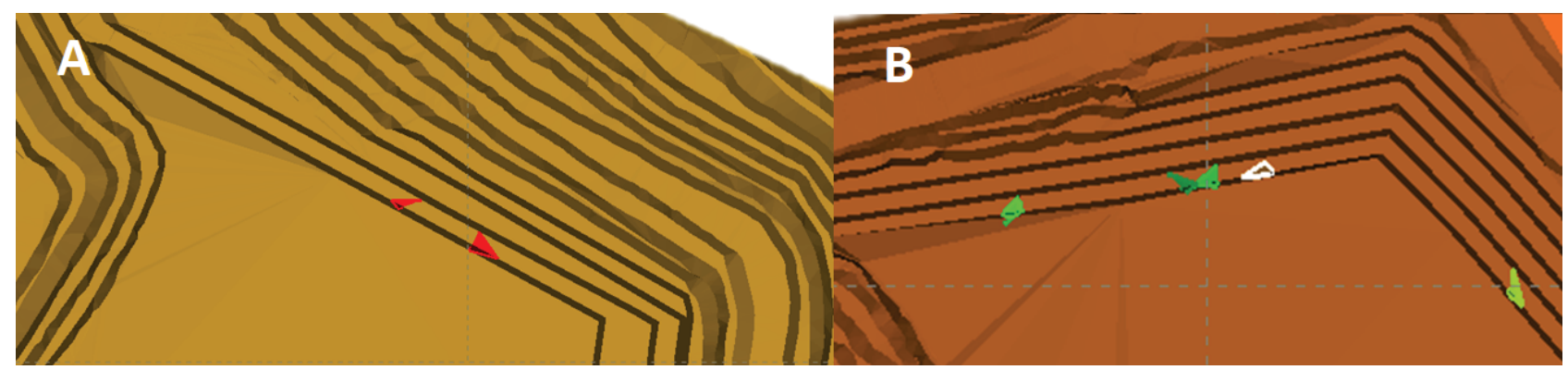

Figure 10 Blocks with Safety Factor under 1.5 and Probability of Failure greater than $20 \%$

The geotechnical team recommended that the Operations Department extract these instabilities with the shovels when they are removing the material from the bench implicated after the blasting. 
When Operations were doing this procedure, they could notice that those instabilities were formed as TIMining Tangram predicted (Figures 11 and 12).
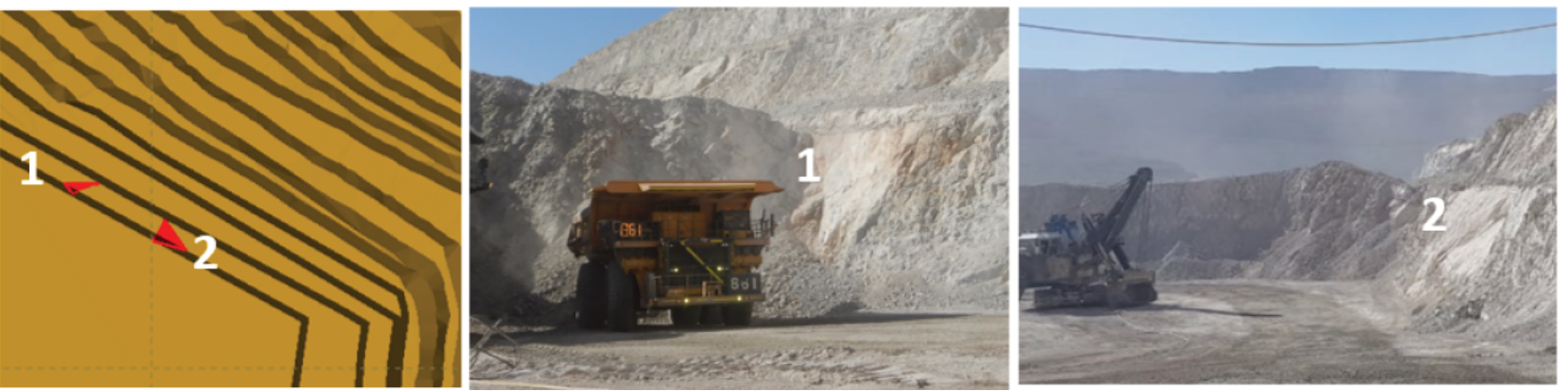

Figure 11 Unstable blocks of design surface A. 1: Plane Fault (0.2 Ktonne), Safety Factor (0.98) and Failure Probability (24\%). 2: Plane Fault (1.7 Ktonne), Safety Factor (0.75) and Failure Probability (83\%)
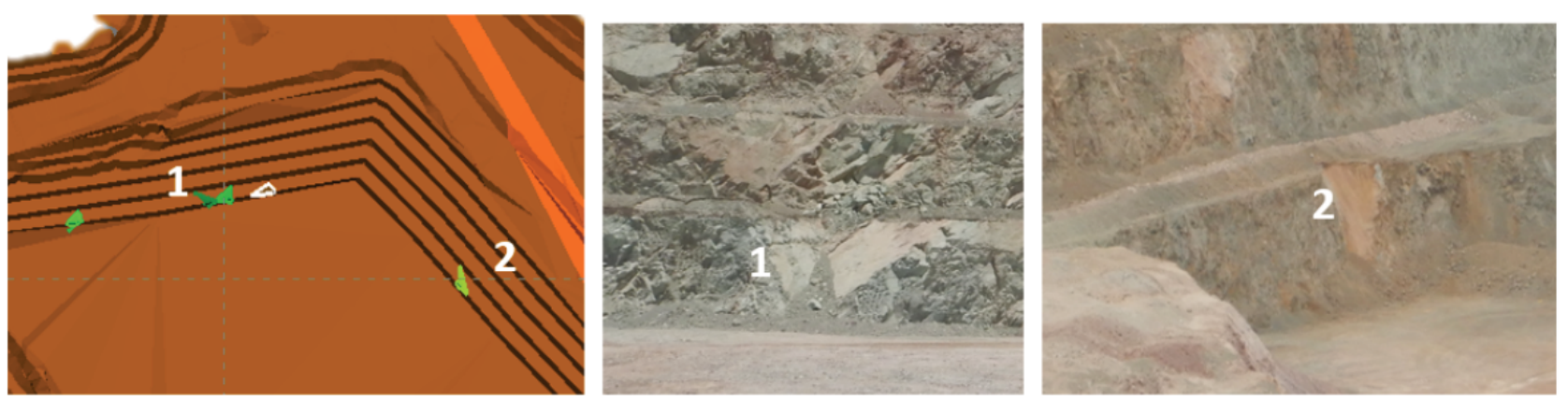

Figure 12 Unstable blocks of design surface B. 1: Plane Fault (1.1 Ktonne), Safety Factor (0.45) and Failure Probability (99\%). 2: Plane Fault (1.7 Ktonne), Safety Factor (0.83) and Failure Probability (74\%)

\section{Conclusion}

TIMining Tangram can be used to support geotechnical monitoring systems with early identification of structural instabilities for a focused control on those areas and preventing operational disruption. It also can be used to detect potential instabilities on real and design surfaces, in order to reduce the number of accidents and geotechnical events and increase the operational continuity and the mining plans reliability.

The early identification of structural instabilities can prevent accidents and saves large amounts of work, increasing the reliability of mine plans. Preventive measures can be taken long before mining operations start if instabilities are identified during design.

The availability of a tool to identify instabilities using actual data from the mine site has allowed the geotechnical department to structurally analyse the alert zone, enabling the performance of such analysis using the actual pit surface and structures in the mine, removing the need to simulate surfaces or structures and thus providing an accurate diagnosis.

This analysis has enabled the estimation of the identified instability's size and the preparation of mitigation plans including bench-to-bench removal of the instability, which have been included in the mining plan by the Planning Department. Removal of the instability has been considered successful, despite the second alert by the monitoring system prevented its completion, as the removed material was enough for the rock mass to settle and decrease its deformation rate.

In addition, the short-term mining plan has been adjusted to consider the time and work required to support the removal of instabilities, which results in a realistic estimation of the ability to carry out future work, thus increasing the reliability of the mining plan. 


\section{References}

Dimitrakopoulos, R, Farrelly, T \& Godoy, M 2002, 'Moving forward from traditional optimization: grade uncertainty and risk effects in open-pit design', Transactions of the Institution of Mining and Metallurgy, Mining Technology, vol. A, no. 111, pp. 82-88. Goodman, R \& Shi, G 1985, Block Theory and its Application to Rock Engineering, Prentice-Hall, London. Lane, K 1988, The Economic Definition of Ore, Mining Journal Books Ltd, London, pp. 147-266.

TIMining Tangram 2019, TIMining Tangram - Software for Detection of Instabilities with Structural Control, computer software, Santiago, www.timining.com 